\title{
Composition and band structure of the native oxide nanolayer on the ion beam treated surface of the GaAs wafer
}

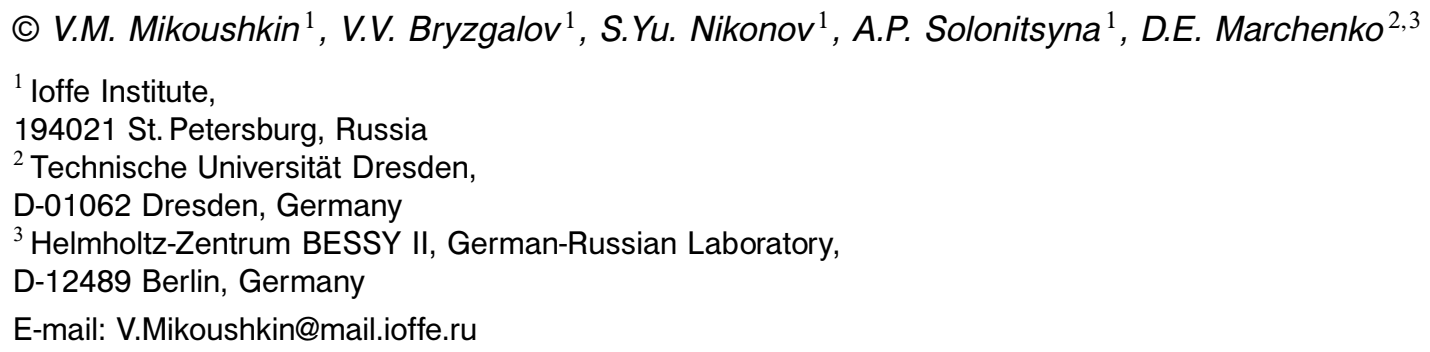

Detailed information on GaAs oxide properties is important for solving the problem of passivating and dielectric layers in the GaAs-based electronics. The elemental and chemical compositions of the native oxide layer grown on the atomically clean surface of an $n-\mathrm{GaAs}(100)$ wafer etched by $\mathrm{Ar}^{+}$ions have been studied by synchrotron-based photoelectron spectroscopy. It has been revealed that the oxide layer is essentially enriched in the $\mathrm{Ga}_{2} \mathrm{O}_{3}$ phase which is known to be a quite good dielectric as compared to $\mathrm{As}_{2} \mathrm{O}_{3}$. The gallium to arsenic ratio reaches the value as high as $[\mathrm{Ga}] /[\mathrm{As}]=1.5$ in the course of oxidation.The Ga-enrichment occurs supposedly due to diffusion away of As released in preferential oxidation of $\mathrm{Ga}$ atoms. A band diagram was constructed for the native oxide nanolayer on the $n$-GaAs wafer. It has been shown that this natural nanostructure has features of a $p-\mathrm{n}$ heterojunction.

\section{Acknowledgements}

This work was supported by the Russian Science Foundation (Project No 17-19-01200). The authors also thank Helmholtz Zentrum Berlin (HZB) for the allocation of synchrotron radiation beamtime at the Russian-German Laboratory of BESSY II Helmholtz Zentrum Berlin for the support in the synchrotron radiation experiments. 